\title{
Characterization and fine mapping of the rice blast resistance gene Pia
}

\author{
ZENG XiaoShan ${ }^{1 \dagger}$, YANG XianFeng $^{1 \dagger}$, ZHAO ZhengHong $^{2}$, LIN Fei $^{1}$, \\ WANG Ling ${ }^{1} \&$ PAN QingHua ${ }^{1 *}$ \\ ${ }^{1}$ Laboratory of Plant Resistance and Genetics, College of Natural Resources and Environment Sciences, \\ South China Agricultural University, Guangzhou 510642, China; \\ ${ }^{2}$ Hunan Agricultural University, Changsha 410128, China
}

Received October 11, 2010; accepted December 8, 2010; published online March 4, 2011

\begin{abstract}
Blast, caused by Magnaporthe oryzae, is one of the most widespread and destructive diseases of rice. Breeding durable resistant cultivars (cvs) can be achieved by pyramiding of various resistance $(R)$ genes. Pia, carried by cv. Aichi Asahi, was evaluated against 612 isolates of M. oryzae collected from 10 Chinese provinces. The Pia gene expresses weak resistance in all the provinces except for Jiangsu. Genomic position-ready marker-based linkage analysis was carried out in a mapping population consisting of $800 \mathrm{~F}_{2}$ plants derived from a cross of Aichi Asahi $\times$ Kasalath. The locus was defined in an interval of approximately $90 \mathrm{~kb}$, flanked by markers A16 and A21. Four candidate genes (Pia-1, Pia-2, Pia-3, and Pia-4), all having the R gene conserved structure, were predicted in the interval using the $\mathrm{cv}$. Nipponbare genomic sequence. Four candidate resistance gene (CRG) markers (A17, A25, A26, and A27), derived from the four candidates, were subjected to genotyping with the recombinants detected at the flanking markers. The first three markers completely co-segregated with the Pia locus, and the fourth was absent in the Aichi Asahi genome and disordered with the Pia locus and its flanking markers, indicating that the fourth candidate gene, Pia-4, could be excluded. Co-segregation marker-based genotyping of the three sets of differentials with known $R$ gene genotypes revealed that the genotype of A26 (Pia-3) perfectly matched the $R$ gene genotype of Pia, indicating that Pia-3 is the strongest candidate gene for Pia.
\end{abstract}

rice blast, resistance gene, Pia, fine mapping, genotype

Citation: Zeng X S, Yang X F, Zhao Z H, et al. Characterization and fine mapping of the rice blast resistance gene Pia. Sci China Life Sci, 2011, 54: 372-378, doi: $10.1007 / \mathrm{s} 11427-011-4154-1$

Rice blast disease, caused by Magnaporthe oryzae (T. T. Hebert) M. E. Barr (anamorph Pyricularia grisea Sacc.), threatens the sustainable production of rice [1]. The use of resistant cultivars (cvs) is one of the most effective and economical ways to mitigate disease losses [1,2]. However, genetic resistance of rice cvs imparted by a single resistance $(R)$ gene is commonly short-lived because of the occurrence of new virulent race(s) adopting the $R$ gene $[3,4]$. By con-

$\dagger$ Contributed equally to this work

*Corresponding author (email: panqh@scau.edu.cn) trast, stacking of multiple $R$ genes into individual cvs has been advocated to achieve durable blast resistance [5-8]. On the other hand, the majority of plant $R$ genes belong to the large NBS-LRR (nucleotide-binding site-leucine-rich repeat) gene family, which aids the search for candidate $R$ gene(s) in defined genomic regions [1,9]. This type of $R$ gene can be subdivided into stronger and weaker effect $R$ genes $[6,10]$. Combinations of stronger and weaker effect $R$ genes would favor a broader resistance spectrum and a higher level of resistance [7,10,11]. Therefore, the identification and utilization of weaker effect $R$ genes will signifi- 
cantly improve the durability of genetic resistance in new cvs.

More than 80 major blast $R$ genes have been identified to date $[12,13]$, and 13 were identified in Japanese differential lines. Among these, Pik-s, Pia, and Pii are weaker effect $R$ genes carried by the native Japanese cvs Shin2, Aichi Asahi, and Fujisaka 5, respectively [6,14]. Pia, which displays a narrow spectrum resistance and is widely present in Japanese rice cvs $[6,15,16]$, is located on the short arm of rice chromosome 11 [17-19]. For better utilization of the Pia gene in China, its resistance to the fungal pathogen populations collected from various regions in China was characterized and its locus was finely mapped in a larger mapping population using genomic position-ready markers.

\section{Materials and methods}

\subsection{Isolates}

A total of 612 Chinese blast isolates were collected from Guangdong (GD, 60 isolates), Fujian (FJ, 40 isolates), Hunan (HN, 40 isolates), Guizhou (GZ, 60 isolates), Yunnan (YN, 43isolates), Sichuan (SC, 66 isolates), Jiangsu (JS, 72 isolates), Liaoning (LN, 108 isolates), Jilin (JL, 60 isolates), and Heilongjiang (HLJ, 63 isolates), and used to compare the specific reaction patterns and resistance frequencies. $\mathrm{F}_{2}$ plants derived from a cross of Aichi Asahi×Kasalath were inoculated with a stable Magnaporthe grisea isolate, CHL272, which is avirulent on Aichi Asahi and virulent on Kasalath. Inoculations and disease evaluations were conducted in greenhouses, as previously described by Pan et al. [20].

\subsection{Resistance spectrum analysis}

Resistance performance of three Japanese differential cvs, Shin2, Aichi Asahi, and Fujisaka 5, was assessed using the 10 populations mentioned above. Resistance evaluation was represented by the resistance frequency: the number of avirulent isolates compared with the total number of isolates examined for a rice cultivar in every population. The resistance frequencies were generated after statistical analysis in Microsoft Excel (Figure 1).

\subsection{Mapping population construction}

The $\mathrm{F}_{2}$ plants derived from a cross of Aichi Asahi×Kasalath were used for resistance segregation analysis and gene mapping. Seedlings of the parents and their progeny were grown in a greenhouse and then challenged with the isolate CHL272 at the four- to five-leaf stage. The inoculation and disease evaluation were conducted in accordance with the method outlined in Pan et al. [20]. The leaves of seedlings were sampled and frozen.

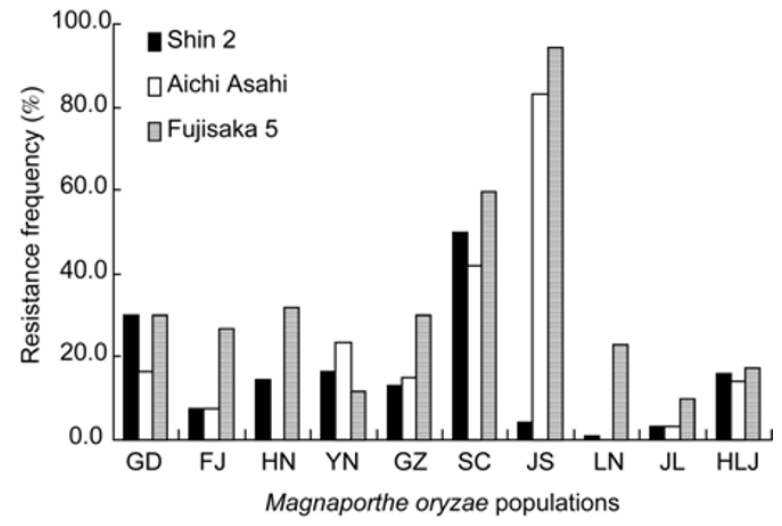

Figure 1 Resistance characterization of three Japanese differential cultivars in the 10 Magnaporthe oryzae populations collected in China.

\subsection{DNA extraction and resistant and susceptible pool construction}

Total DNA was extracted from frozen leaves of the seedlings by the CTAB method. Contrast DNA pools were assembled by mixing equimolar amounts of DNA from either 10 resistant or 10 susceptible $F_{2}$ individuals. The bulkedsegregant analysis (BSA) was carried out to identify molecular markers putatively associated with the $R$ gene locus.

\subsection{Linkage analysis}

The Pia locus was mapped in three steps: recombinant screening, fine mapping, and co-segregation analysis [21]. First, simple sequence repeat (SSR) markers on the short arm of rice chromosome 11 were used for BSA analysis [22]. Those markers that produced a differential banding pattern from the resistant and susceptible pools were then genotyped in the whole mapping population by screening for recombinants occurred at the respective marker loci linked to the locus. For constructing the linkage map of the locus, sequences of the linked markers were downloaded from GRAMENE (http://www.gramene.org) and placed on the reference sequence of cv. Nipponbare (http://www.ncbi. nlm.nih.gov/blast). Second, an additional set of SSR markers, in addition to sequence-tagged site (STS) markers, were developed in the defined region for further linkage analysis [23]. Primer pairs were designed using Primer Premier 5.0 (http://primerbiosoft.com). The new markers, which showed clear polymorphisms between the parents, were then applied to a set of recombinant progeny. The physical map covering Pia gene locus was constructed, in silico, using the genomic position-ready markers $[21,23]$. Finally, a set of candidate resistance gene (CRG) markers was developed in the target region on the basis of open reading fragments of candidate $R$ genes with an NBS-LRR structure, as annotated by the RiceGAAS (http://rgp.dna.affrc.go.jp) software. CRG markers that co-segregated with the Pia locus were identified by the recombinants detected at the 
flanking marker loci. All the markers are listed in Table 1.

\subsection{Co-segregation markers-based genotyping}

To further determine candidate gene(s) for Pia, three sets of differential lines (mostly carrying known $R$ genes; Table 2 ), developed in Japan, China, and the International Rice Research Institute (IRRI) were genotyped with the co-segregation markers.

\section{Results}

\subsection{Resistance characterization}

Resistance performances of three Japanese differential cvs, Shin2, Aichi Asahi and Fujisaka 5 in the 10 M. grisea populations are shown in Figure 1. All the three cvs showed moderate levels of resistance to the SC population, and both
Aichi Asahi and Fujisaka 5 showed a higher level of resistance to the JS population. For the other eight populations, all the three cvs showed lower levels of resistance.

\subsection{Mapping population construction}

The segregation between resistance and susceptibility of a total of $924 \mathrm{~F}_{2}$ individuals was consistent with $3 \mathrm{R}: 1 \mathrm{~S}$ (resistant/susceptible, $\left.686 / 238\left(\chi_{\mathrm{c}}^{2}=0.24, P>0.6\right)\right)$, indicating that Aichi Asahi carries a monogenic dominant $R$ gene, which confers resistance to the isolate CHL272. Therefore, a mapping population, consisting of 585 resistant and 215 susceptible individuals with extreme phenotypes selected from the $924 \mathrm{~F}_{2}$ progeny, were carried forward (some susceptible plants died).

\subsection{Confirmation of the Pia gene locus}

Pia, carried by Aichi Asahi, was previously mapped on the

Table 1 Experimental details of PCR-based markers used for linkage analysis of Pia

\begin{tabular}{|c|c|c|c|c|c|}
\hline Marker $^{a)}$ & Type & Primer sequence $\left(5^{\prime}-3^{\prime}\right)^{\mathrm{b})}$ & Genomic position $(b p)^{c)}$ & Annealing temperature $\left({ }^{\circ} \mathrm{C}\right)^{\mathrm{d})}$ & Expected size (bp) \\
\hline \multicolumn{6}{|c|}{ Recombinants screening } \\
\hline RM332 & SSR & $\begin{array}{l}\text { F: GCGAAGGCGAAGGTGAAG } \\
\text { R: CATGAGTGATCTCACTCACCC }\end{array}$ & $\begin{array}{l}2827519-2827536 \\
2827669-2827689\end{array}$ & 55 & 171 \\
\hline RM167 & SSR & $\begin{array}{l}\text { F: GATCCAGCGTGAGGAACACGT } \\
\text { R: AGTCCGACCACAAGGTGCGTTGTC }\end{array}$ & $\begin{array}{l}4060683-4060463 \\
4060360-4060337\end{array}$ & 58 & 147 \\
\hline RM441 & SSR & $\begin{array}{l}\text { F: CGATGACACACAATTCACACA } \\
\text { R: CACATAGGCAAGTCATTCT }\end{array}$ & $\begin{array}{l}6068480-6068461 \\
6068339-6068321\end{array}$ & 58 & 160 \\
\hline RM536 & SSR & $\begin{array}{l}\text { F: TCTCTCCTCTTGTTTGGCTC } \\
\text { R: ACACACCAACACGACCACAC }\end{array}$ & $\begin{array}{l}9035072-9035091 \\
9035295-9035314\end{array}$ & 55 & 243 \\
\hline \multicolumn{6}{|c|}{ Fine mapping } \\
\hline A7 & SSR & $\begin{array}{l}\text { F: GAATAGTCTTACCCCCCCC } \\
\text { R: CCTTGTAAATTCCCCTTTGTTG }\end{array}$ & $\begin{array}{l}6245047-6246065 \\
6246170-6246191\end{array}$ & 60 & 145 \\
\hline A 15 & SSR & $\begin{array}{l}\text { F: CTCCTCCATTTTTTCCCATCC } \\
\text { R: GTGGAGGAGCCAAGAACAG }\end{array}$ & $\begin{array}{l}6391668-6391688 \\
6391775-6391793\end{array}$ & 64 & 126 \\
\hline A21 & STS & $\begin{array}{l}\text { F: CAGACGGTTTCAGAACGAGG } \\
\text { R: CAGAACAAGACAGCATAACCC }\end{array}$ & $\begin{array}{l}6583046-6583062 \\
6584044-6584064\end{array}$ & 58 & 1022 \\
\hline A9 & SSR & $\begin{array}{l}\text { F: ATAGGGCCCACAAATCACATC } \\
\text { R: GGCCCAATGGACAAATTCTTA }\end{array}$ & $\begin{array}{l}6651922-6651942 \\
6652065-6652085\end{array}$ & 62 & 164 \\
\hline \multicolumn{6}{|c|}{ Co-segregation analysis } \\
\hline A 17 & CRG & $\begin{array}{l}\text { F: GCAACGGATACGGAGGCAAT } \\
\text { R: GCTTTTCTTAGCAATGTCTGTG }\end{array}$ & $\begin{array}{l}6518740-6518721 \\
6517729-6517708\end{array}$ & 58 & 1033 \\
\hline A25 & CRG & $\begin{array}{l}\text { F: TAAAAATGAGGTTGGGAGTC } \\
\text { R: GTTCTTAGCAATGATGTCCTC }\end{array}$ & $\begin{array}{l}6523045-6523064 \\
6523934-6523954\end{array}$ & 58 & 908 \\
\hline A26 & CRG & $\begin{array}{l}\text { F: CTGAAGAGGATGGTGGAGGA } \\
\text { R: ATTGTAAACATCCCCATCTG }\end{array}$ & $\begin{array}{l}6528288-6528269 \\
6527480-6527461\end{array}$ & 58 & 828 \\
\hline $\mathrm{A} 27^{\mathrm{e})}$ & CRG & $\begin{array}{l}\text { F: CTGGGAGTATCTACAGAAAAAG } \\
\text { R: CTCTGGAAAAGCACTGGTAAT }\end{array}$ & $\begin{array}{l}6538262-6538283 \\
6539453-6539473\end{array}$ & 58 & 1212 \\
\hline
\end{tabular}

a) Markers used for recombinant screening, fine mapping, and co-segregation analysis are grouped. Markers prefixed with RM were adopted from GRAMENE (http://www.gramene.org) and those prefixed with A were developed in the present study. b) F, forward; R, reverse. c) The genomic position of each marker on the short arm of rice chromosome 11 was determined by BLASTN analysis against the reference sequence of cv. Nipponbare (http://ncbi.nlm.nih.gov/blast). d) All PCR runs began with one cycle at $94^{\circ} \mathrm{C}$ for $3 \mathrm{~min}$, followed by 35 cycles at $94^{\circ} \mathrm{C}$ for $30 \mathrm{~s}, 55-64^{\circ} \mathrm{C}$ for $30 \mathrm{~s}$, and $72^{\circ} \mathrm{C}$ for $1-1.5 \mathrm{~min}$; with a final extension at $72^{\circ} \mathrm{C}$ for $7 \mathrm{~min}$. Amplicons were separated by electrophoresis on $3 \%$ and $6 \%$ polyacrylamide gels, separately. e) A27 was a dominate marker absent in Aichi Asahi and did not exhibit linkage with markers flanking Pia (Figure 2). 
short arm of rice chromosome 11 [17-19]; therefore, nine SSR markers (RM286, RM558B, RM332, RM167, RM552, RM441, RM479, RM202, and RM536), which are evenly distributed on the short arm, were selected for BSA analysis. Four polymorphic markers were used for linkage analysis in the whole mapping population. The results showed that there were 145,102 , and 15 recombinants which overlapped with loci RM332, RM167, and RM441, respectively, indicating that these three markers lie on the same side of the Pia locus. On the other side, 116 distinct recombinants, except for \#290, were detected at RM536. Thus, the Pia locus was flanked by these four markers (on the telomeric side, RM332, RM167, and RM441; on the centromeric side, RM536) (Figure 2(B)). To further confirm the recombinants on the centromeric side, a new SSR marker, A6, was developed for screening the recombinants in the whole mapping population. As expected 29 recombinants overlapping with RM536 were detected for this marker. Thus, Pia was mapped to a segment flanked by RM441 and A6, which covers $1050 \mathrm{~kb}$ (Figure 2(B)). A total of 44 recombinants detected at RM441 and A6 were subjected to fine mapping.

\subsection{Fine mapping of the Pia gene locus}

To narrow the region spanning Pia locus, four SSR markers (A7, A9, A15, and A16) and one STS marker (A21) were developed in the region flanked by RM441 and A6. On the telomeric side, 11, eight, and three recombinants derived from RM441 were detected at A7, A15, and A16, respectively. Five and one recombinant(s) originating from A6 were detected at A9 and A21, respectively, on the centromeric side. The location of Pia was thereby narrowed to a $90 \mathrm{~kb}$ region flanked by A16 and A21 (Figure 2(B)). The correlation between phenotypes and genotypes of the three

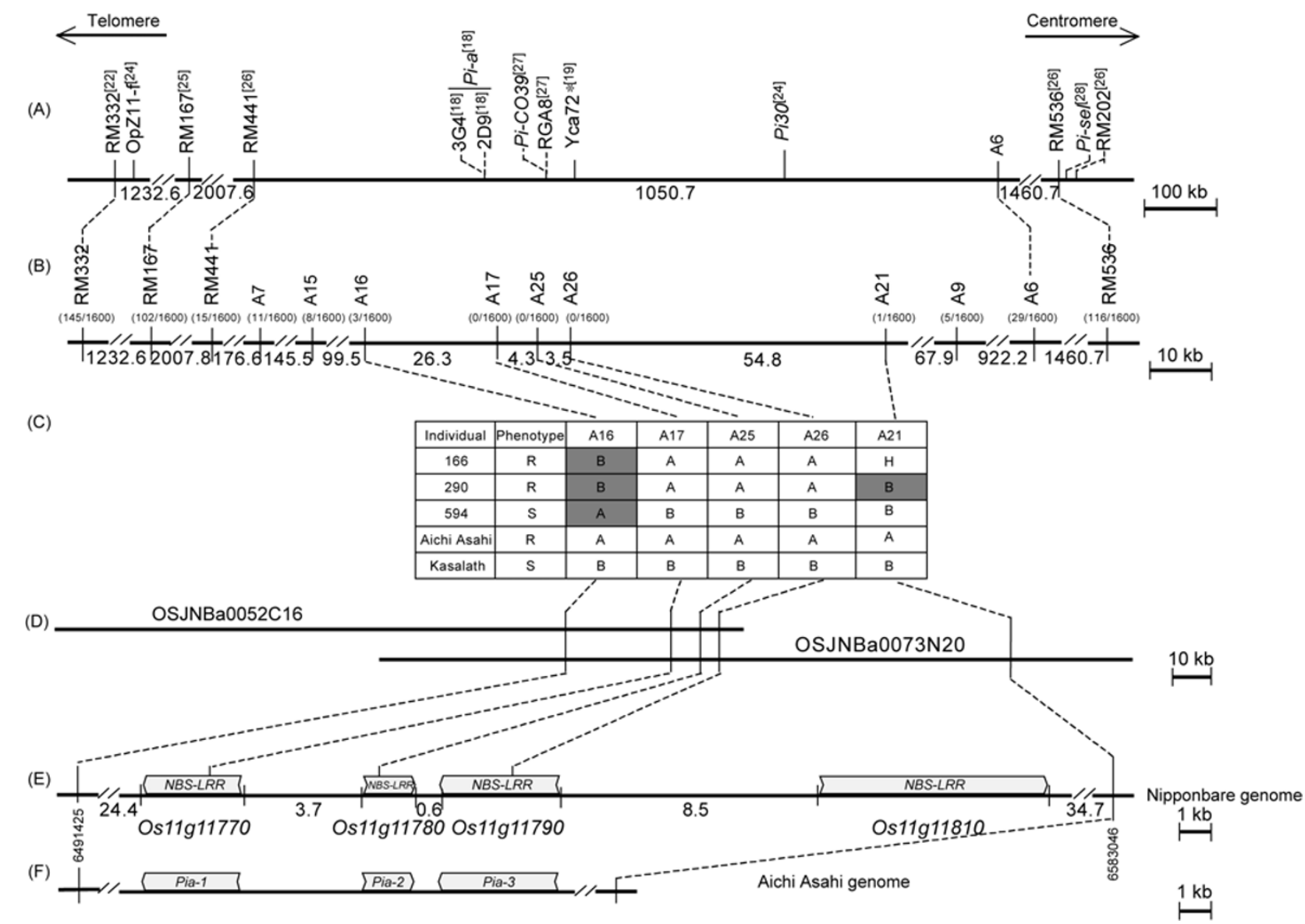

Figure 2 High resolution physical map of the Pia locus. (A) An integrated physical map of Pia, including three blast resistance genes neighbored to Pia, i.e., Pi30, Pi-CO39, and Pi-sel. Map positions were inferred from previous investigations [18,19,22,24-28]. (B) An in silico localized physical map of Pia based on this study. The numbers below the map represent physical distance of the cv. Nipponbare genomic sequence. The numbers in parentheses are the numbers of recombinants detected between marker loci and Pia. (C) Correlation between phenotypes and genotypes of key recombinants and parents at the loci co-segregated with Pia. R, resistance; S, susceptible; A, homozygous allele of resistance parent Aichi Asahi; B, homozygous allele of susceptible parent Kasalath; H, heterozygous alleles of both parents. Genotypes with shadow represent recombinants. (D) Contig map spanning the Pia locus. The short horizontal lines represent BAC clones of cv. Nipponbare. The vertical lines denote the positions of the respective markers. (E) Four candidate genes predicted as NBS-LRR in cv. Nipponbare genome were identified in the defined region by RiceGAAS (http://ricegaas.dna.affrc.go.jp). The numbers below the map are physical distances between genes. The numbers on both ends of the map denote the position of markers flanking Pia locus on Nipponbare genomic sequence.

(F) The structural features and candidate genes at the Pia locus in the cv. Aichi Asahi genome. 
key recombinants and parents at the loci of A16 and A21 are listed in Figure 2(C).

\subsection{Co-segregation analysis of candidate genes for Pia}

The target region defined by A16 and A21 was located on two BAC clones, OSJNBa0052C16 and OSJNBa0073N20, of reference cv. Nipponbare, which cover 6491425$6583046 \mathrm{bp}$ (Figure 2(D)). According to the annotation of these two BAC clones by RiceGAAS, four genes, Os11g11770, Os11g11780, Os11g11790, and Os11g11810 encoding NBS-LRR domains, were identified as candidate genes of Pia in the target region of the cv. Nipponbare genome. To obtain additional markers that completely cosegregate with the Pia locus, additional CRG markers based on the coding sequence of every candidate gene were developed for the final round of linkage analysis. Three CRG markers (A17, A25 and A26) derived from Os11g11770, Os11g11780, and Os11g11790, respectively, completely co-segregated with the Pia locus (Figure 2(C) and (E)). However, A27, derived from Os11g11810, which was dominant, is present in the susceptible cvs. Kasalath and Nipponbare and absent in the resistant parent cv. Aichi Asahi. Recombinant genotyping showed that the linkage relationship between A27 and the Pia loci was disordered. To carefully rule out os 11 g11810 in the cv. Aichi Asahi genome, three additional markers developed on both sides of $\operatorname{os} 1 \mathrm{lg} 11810$ were used for linkage analysis. The results indicated that recombinants detected on these three marker loci were also disordered (data not shown). Thus, the data indicate that there are two genome types in the Pia-related region between Nipponbare and Aichi Asahi, and in the latter, the fourth candidate gene $O \operatorname{sl} 1 \mathrm{~g} 11810$ has been lost and/or shifted (Figure 2(E) and (F)).

Table 2 Genotyping of three candidate genes with the co-segregating markers to the Pia locus in three sets of rice blast differential lines

\begin{tabular}{|c|c|c|c|c|c|}
\hline \multirow{2}{*}{ Differential } & \multirow{2}{*}{ Resistance gene $^{a)}$} & \multirow{2}{*}{ Subspecies } & \multicolumn{3}{|c|}{ Marker $^{\text {b) }}$} \\
\hline & & & A17 & A25 & A26 \\
\hline \multicolumn{6}{|l|}{ Japanese differentials } \\
\hline Shin 2 & Pik-s, Pish & Japonica & $\mathrm{B}$ & A & B \\
\hline Aichi Asahi & Pia & Japonica & A & A & A \\
\hline Fujisaka 5 & Pii, Pik-s & Japonica & $\mathrm{B}$ & A & B \\
\hline Kusabue & Pik, Pish & Japonica & $\mathrm{B}$ & A & B \\
\hline Fukunishiki & Piz, Pish & Japonica & $\mathrm{B}$ & A & B \\
\hline K 1 & Pita & Japonica & B & A & B \\
\hline Pi No.4 & Pita-2, Pish & Japonica & B & A & B \\
\hline Toride 1 & Piz-t, Pish & Japonica & A & A & A \\
\hline K 60 & Pik-p, Pish & Japonica & B & A & B \\
\hline BL 1 & Pib, Pish & Japonica & $\mathrm{B}$ & A & B \\
\hline K 59 & Pit, Pik-s & Japonica & B & A & B \\
\hline Tetep & Pi1, Pi5, Pi4-b & Indica & A & A & A \\
\hline Zhenglong 13 & Unknown & Indica & B & A & B \\
\hline Sifeng 43 & Pia, Pit/Pib & Indica & A & A & A \\
\hline Dongnong 363 & Pia, Pik & Japonica & $\mathrm{A}$ & A & A \\
\hline Kando 51 & $P i k$ & Japonica & A & A & B \\
\hline Huojiang 18 & Pia, Pii & Japonica & A & A & A \\
\hline LTH & None & Japonica & B & A & B \\
\hline \multicolumn{6}{|l|}{ IRRI differentials } \\
\hline $\mathrm{CO} 39$ & Pia, PiCO39 & Indica & A & A & A \\
\hline C101LAC & Pia, Pil & Indica & A & A & A \\
\hline C101A51 & Pia, Pi2 & Indica & A & A & A \\
\hline C101PKT & Pia, Pi4- $a$ & Indica & A & A & A \\
\hline C104PKT & Pia, Pi3 & Indica & A & A & A \\
\hline
\end{tabular}

a) Resistance genes in Japanese, Chinese, and IRRI differential lines were quoted from Imbe et al. [14], Kiyosawa and Ling [29], Inukai et al. [30], and Kobayashi et al. [31].

b) The co-dominant markers A17, A25, and A26 corresponded to the candidate genes Pia-1, Pia-2, and Pia-3, respectively (also see Figure 2). "A" denotes the genotype of the resistant parental cv. Aichi Asahi; "B" denotes the susceptible parental cv. Kasalath. Genotypes in the box were used to deduce Pia-3 as the most promising candidate gene for Pia. 


\subsection{Genotyping of differential lines}

To further confirm candidate gene(s) for Pia, three sets of rice blast differentials, mostly carrying known $R$ gene(s), were used to genotype with the co-segregation markers derived from each of the three candidate genes (Table 2, Figure 2). The genotype of marker A25 was identical with cv. Aichi Asahi in all the differentials tested, indicating that Os11g11780 might not be the functional Pia gene. As for the other two candidate genes, genotypes of marker A26 completely corresponded to the genotypes of resistance, and those of marker A17 did not. When we focused on the four differentials in the box (Figure 2), it became clear that only the genotypes of marker A26 were consistent with the genotypes of their $R$ genes (Table 2). It could be concluded that Os11g11790 (A26) is the most promising candidate gene for Pia, rather than Os11g11770 (A17). This was supported by the identical genotype of marker A26 in the IRRI differential lines, because the recurrent parental cv. CO39 for these near-isogenic lines was confirmed to carry the Pia gene [31]. Intriguingly, two differentials, Toride 1 and Tetep, which were not found to have the Pia gene in previous studies, should also carry the Pia gene, based on the genotyping results obtained in the present study (Table 2).

\section{Discussion}

Rice blast is a tricky disease in rice production, because genetic resistance of newly developed cvs is commonly rendered ineffective within a few years after release [5,32]. One long-term strategy for the control of this disease may depend on the durable genetic resistance conferred by $R$ gene stacking [6-8,32]. The resistance of $R$ genes can be divided into stronger and weaker effects $[6,10]$. The $R$ genes carried by the Japanese differentials, Pik, Pik-m, and Pik-p, exerted stronger effects in most southern regions of China [21]; however, Pik-s, Pish, Pia, and Pii displayed weaker effects in these regions (Figure 1). Results from earlier studies indicated that these last four $R$ genes, which are ignored by breeders, could have important roles after pyramiding with other $R$ genes, whether weaker or stronger $R$ genes $[6,7,10,11]$. It is necessary to assemble better combinations of $R$ genes that possess economic, effective, and durable resistance without damaging the other agronomic traits of the crop.

Rice is a model crop with two available reference sequences of two subspecies. The genomic region of a target gene, in which the target gene is isolated via an approach called map-based cloning in silico, can be rapidly defined through chromosome-walking with genomic position-ready markers, developed from the available reference sequences. Such a strategy could save a great deal of time and labor because there is no need to construct an artificial chromosome library, and it also could overcome the mapping diffi- culty because of any complex genetic exchange and genomic structure dynamics (if any) that have occurred in the target region $[2,23,33,34]$. On the other hand, genomic regions of $R$ genes are particularly dynamic and complex because the long-term co-evolution with $A V R$ genes of pathogens [2,4,35-37]. A greater genomic difference was also detected in the Pia-surrounding region between the resistance donor cv. Aichi Asahi and the susceptible reference cv. Nipponbare, which resulted in the loss of the fourth candidate gene Osl1g11810 in the Aichi Asahi genome (Figure 2). The isolation of Pia will also provide insight into the molecular evolution of $R$ genes.

Many plant $R$ genes are clustered in special genomic regions, and encode highly conserved motifs and structures, such as the NBS-LRR proteins $[4,9,33,34,38]$. To date, 15 major blast $R$ genes have been cloned, 13 of which belong to the large NBS-LRR gene family [2,38]. In the present study, the Pia locus was defined into a region with a cluster of four NBS-LRR genes, based on the reference sequence of cv. Nipponbare (Figure 2(E)). Among these four candidate genes, Pia-3 (Os11g11790) was identified as the most promising candidate for Pia via CRG marker-based genotyping (Table 2). Sequence alignment analysis revealed that Pia-3 has a high degree of similarity to the $R$ gene $R P M 1$ of Arabidopsis thaliana (data not shown). Interestingly, two ESTs (expressed sequence tags; 2D9 and 3G4; Figure 2(A)), generated from a double haploid (DH) mapping population derived from the cross of IR64 and Azucena, are tightly linked to Pia locus [18]. Furthermore, the coding sequences of both ESTs are also highly similar to RPM1. The present study also validates the approach of candidate gene(s) by CRG marker-based genotyping [2,33,34]. We have embarked on the identification of the functional Pia gene via forward genetic complementation.

This work was supported by the National Transgenic Research Projects (Grant No. 2009ZX08009-023B), the National Basic Research Program of China (Grant No. 2011CB1007), and the National Commonweal Specialized Research Project (Grant No. 200803008). Materials used in the present research were obtained from our own collection, as well as by exchange of materials with colleagues. We thank our colleagues for their enthusiasm and help.

1 Jeung J, Kim B, Cho Y, et al. A novel gene, Pi4O(t), linked to the DNA markers derived from NBS-LRR motifs confers broad spectrum of blast resistance in rice. Theor Appl Genet, 2007, 115: 1163-1177

2 Zhai C, Lin F, Dong Z, et al. The isolation and characterization of $P i k$, a rice blast resistance gene which emerged after rice domestication. New Phytol, 2011, 189: 321-334

3 Wang G, Mackill D, Bonman J, et al. RFLP mapping of genes conferring complete and partial resistance to blast in a durably resistant rice cultivar. Genetics, 1994, 136: 1421-1434

4 Zhou B, Dolan M, Sakai H, et al. The genomic dynamics and evolutionary mechanism of the Pi2/Pi9 locus in rice. Mol Plant Microbe Interact, 2007, 20: 63-71

5 Ou S. Pathogen variability and host resistance in rice blast disease. Ann Rev Phytopathol, 1980, 18: 167-187

6 Kiyosawa S. Studies on genetics and breeding of blast resistance in 
rice (in Japanese). Misc Publ Natl Inst Agric Sci, 1974, 1: 1-58

7 Hittalmani S, Parco A, Mew T, et al. Fine mapping and DNA marker-assisted pyramiding of the three major genes for blast resistance in rice. Theor Appl Genet, 2000, 100: 1121-1128

8 Fuentes J, Corrrea-Victoria F, Escobar F, et al. Identification of microsatellite markers linked to the blast resistance gene $P i-1(t)$ in rice. Euphytica, 2008, 160: 295-304

9 Takken F, Tameling W. To nibble at plant resistance proteins. Science, 2009, 324: 744-746

10 Adillah Tan M. Genetic mapping and pyramiding of resistance genes in potato. Dissertation for the Doctoral Degree. The Netherlands: Wageningen University, 2008

11 Liu S, Li X, Wang Z, et al. Gene pyramiding to increase the blast resistance in rice (in Chinese). Mol Plant Breed, 2003, 1: 22-26

12 Yang Q, Lin F, Feng S, et al. Recent progress on molecular mapping and cloning of blast resistance genes in Rice (Oryza sativa L.) (in Chinese). Sci Agric Sin, 2009, 42: 1601-1615

13 Ballini E, Morel J, Droc G, et al. A genome-wide meta-analysis of rice blast resistance genes and quantitative trait loci provides new insights into partial and complete resistance. Mol Plant Microbe Interact, 2008, 21: 859-868

14 Imbe $T$, Matsumoto $\mathrm{S}$. Inheritance of resistance of rice varieties to the blast fungus stains virulent to the variety "Reiho" (in Japanese). Jap J breed, 1985, 35: 332-339

15 Yamasaki Y, Kiyosawa S. Studies on inheritance of resistance of rice varieties to blast I. Inheritance of resistance of Japanese varieties to several strains of the fungus (in Japanese). Bull Natl Inst Agric Sci D, 1966, 14: 39-69

16 Fukuta Y, Eboron L, Kobayashi N. Review: Genetic and breeding analysis of blast resistance in elite indica-type rice (Oryza sativa L.) bred in international rice research institute. JAEQ, 2007, 41: 101-114

17 Goto I, Jaw Y, Baluch A. Genetic studies on the resistance of rice plants to the blast fungus. IV. Linkage analysis of four genes, $P i-a$, $P i-k, P i-z$, and Pi-i. Ann Phytopathol Soc Japan, 1981, 47: 252-254

18 Wang Z, Taramino G, Yang D, et al. Rice ESTs with disease-resistance gene- or defense-response gene-like sequences mapped to regions containing major resistance genes or QTLs. Mol Genet Genomics, 2001, 265: 302-310

19 Kwon S, Cho Y, Kim Y, et al. Development of near-isogenic Japonica rice lines with enhanced resistance to Magnaporthe grisea. Mol Cells, 2008, 25: 407-416

20 Pan Q, Hu Z, Takatoshi T, et al. Fine mapping of the blast resistance gene Pi15, linked to Pii, on rice chromosome 9. Acta Bot Sin, 2003, 45: 871-877

21 Wang L, Xu X, Lin F, et al. Characterization of rice blast resistance genes in the Pik cluster and fine mapping of the Pik-p locus. Phytopathology, 2009, 99: 900-905

22 Temnykh S, Park W, Ayres N, et al. Mapping and genome organization of microsatellite sequences in rice (Oryza sativa L.). Theor Appl Gene, 2000, 100: 697-712

23 Liu X, Wang L, Chen S, et al. Genetic and physical mapping of
Pi36(t), a novel rice blast resistance gene located on rice chromosome 8. Mol Genet Genomics, 2005, 274: 394-401

24 Sallaud C, Lorieux M, Roumen E, et al. Identification of five new blast resistance genes in the highly blast-resistant rice variety IR64 using a QTL mapping strategy. Theor Appl Gene, 2003, 106: 794-803

25 Wu K, Tanksley S. Abundance, polymorphism and genetic mapping of microsatellites in rice. Mol Gen Genet, 1993, 241: 225-235

26 Temnykh S, DeClerck G, Lukashova A, et al. Computational and experimental analysis of microsatellites in rice (Oryza sativa L.): Frequency, length variation, transposon associations, and genetic marker potential. Genome Res, 2001, 11: 1441-1452

27 Chauhan R, Farman M, Zhang H, et al. Genetic and physical mapping of a rice blast resistance locus, $\mathrm{Pi}$-CO39(t), that corresponds to the avirulence gene AVR1-CO39 of Magnaporthe grisea. Mol Genet Genomics, 2002, 267: 603-612

28 Wisser R, Sun Q, Hulbert S, et al. Identification and characterization of regions of the rice genome associated with broad-spectrum, quantitative disease resistance. Genetics, 2005, 169: 2277-2293

29 Kiyosawa S, Ling Z. Identification resistance genes of Chinese differential lines by Japanese rice blast isolates (in Chinese). Sci China B, 1984, 1: 44-52

30 Inukai $\mathrm{T}$, Nelson R, Zeigler R, et al. Allelism of blast resistance genes in near-isogenic lines of rice. Phytopathology, 1994, 84: 12781283

31 Kobayashi N, TelebancoYanoria M, Tsunematsu H, et al. Development of new sets of international standard differential varieties for blast resistance in rice (Oryza sativa L.). JARQ, 2007, 41: 31-37

32 Zeigler R, Thome J, Nelson J, et al. Lineage exclusion: A proposal for linking blast population analysis to resistance breeding. In: Proceedings of Rice blast disease CAB International, Los Banos, Laguna, Philippines, 1994. 267-292

33 Lin F, Chen S, Que Z, et al. The blast resistance gene Pi37 encodes an NBS-LRR protein and is a member of a resistance gene cluster on rice chromosome 1. Genetics, 2007, 177: 1871-1880

34 Liu X, Lin F, Wang L, et al. The in silico map-based cloning of Pi36, a rice coiled-coil nucleotide-binding site leucine-rich repeat gene that confers race-specific resistance to the blast fungus. Genetics, 2007, 176: 2541-2549

35 Ashikawa I, Hayashi N, Yamane H, et al. Two adjacent nucleotide-binding site-leucine-rich repeat class genes are required to confer Pikm-specific rice blast resistance. Genetics, 2008, 180: 2267-2276

36 Hayashi K, Yoshida H. Refunctionalization of the ancient rice blast disease resistance gene Pit by the recruitment of a retrotransposon as a promoter. Plant J, 2009, 57: 413-425

37 Lee S, Song M, Seo Y, et al. Rice Pi5-mediated resistance to Magnaporthe oryzae requires the presence of two CC-NB-LRR genes. Genetics, 2009, 181: 1627-1638

38 Yuan B, Zhai C, Wang W, et al. The Pik-p resistance to Magnaporthe oryzae in rice is mediated by a pair of closely linked CC-NBS-LRR genes. Theor Appl Genet, 2011, 122: 1017-1028

Open Access This article is distributed under the terms of the Creative Commons Attribution License which permits any use, distribution, and reproduction in any medium, provided the original author(s) and source are credited. 